\title{
Feeding soy protein isolate and oils rich in omega-3 polyunsaturated fatty acids affected mineral balance, but not bone in a rat model of autosomal recessive polycystic kidney disease
}

\author{
Kaitlin H Maditz', Brenda J Smith², Matthew Miller ${ }^{1}$, Chris Oldaker ${ }^{1}$ and Janet C Tou ${ }^{1 *}$
}

\begin{abstract}
Background: Polycystic kidney disease (PKD), a genetic disorder characterized by multiple cysts and renal failure at an early age. In children, kidney disease is often accompanied by disordered mineral metabolism, failure to achieve peak bone mass, and reduced adult height. Optimizing bone health during the growth stage may preserve against bone loss associated with early renal dysfunction in PKD. Dietary soy protein and omega-3 polyunsaturated fatty acid (n-3 PUFA) have been reported to ameliorate PKD and to promote bone health. The study objective was to determine the bone effects of feeding soy protein and/or n-3 PUFAs in a rat model of PKD.

Methods: Weanling female PCK rats $(n=12$ /group) were randomly assigned to casein + corn oil (Casein $+C O)$, casein + soybean oil (Casein + SO), soy protein isolate + soybean oil (SPI + SO) or soy protein isolate + 1:1 soybean oil:salmon oil blend (SPI + SB) for 12 weeks.

Results: Rats fed SPI + SO diet had shorter $(P=0.001)$ femur length than casein-fed rats. Rats fed SPI + SO and $\mathrm{SPI}+\mathrm{SB}$ diet had higher $(P=0.04)$ calcium $(\mathrm{Ca})$ and phosphorus $(\mathrm{P})$ retention. However, there were no significant differences in femur and tibial $\mathrm{Ca}, \mathrm{P}$ or bone mass between diet groups. There were also no significant difference in bone microarchitecture measured by micro-computed tomography or bone strength determined by three-point bending test between diet groups.
\end{abstract}

Conclusions: Early diet management of PKD using SPI and/or n-3 PUFAs influenced bone longitudinal growth and mineral balance, but neither worsened nor enhanced bone mineralization, microarchitecture or strength.

Keywords: Omega-3 fatty acids, Soy protein, Mineral balance, Long bones, Polycystic kidney disease

\section{Background}

Kidney disease in children is often accompanied by disordered mineral metabolism, decreased adult height, and failure to achieve peak bone mass (PBM) [1,2]. Epidemiological studies demonstrated greater incidence of bone fracture in end stage renal disease patients [3]. Worldwide, the third most common cause of renal failure is polycystic kidney disease which is characterized by multiple cysts, massive enlargement of the kidneys, and loss of renal

\footnotetext{
* Correspondence: janet.tou@mail.wvu.edu

'Division of Animal and Nutritional Sciences, West Virginia University, 1038 Agricultural Sciences Bldg, P.O. Box 6108, Evansdale Campus, Morgantown, West Virginia 26505, USA

Full list of author information is available at the end of the article
}

function [4]. In autosomal dominant polycystic kidney disease (ADPKD), the most prevalent inherited form of polycystic kidney disease, individuals are asymptomatic until the third or fifth decades of life [5]. In the rarer autosomal recessive polycystic kidney disease (ARPKD), disease onset occurs early with more than $50 \%$ of affected children progressing to renal failure in the first decade of life [5,6]. Common strategies for the management of renal disease in childhood include maintaining a healthy body weight and maximizing PBM [7].

Soy protein isolate (SPI) and omega-3 polyunsaturated fatty acids (n-3 PUFAs) are dietary components reported in pre-clinical studies to ameliorate polycystic kidney disease progression and severity [7]. The PCK rat, an 
orthologous model of human ARPKD, has been used in pre-clinical testing of drug treatments [8]. To our knowledge no studies have investigated the effects of dietary SPI on PCK rats. The PCK rat is derived from a spontaneous mutation in Sprague-Dawley rats [9]. Growing female Sprague-Dawley rats fed SPI have been reported to have enhanced bone mineral content (BMC) and bone mineral density (BMD) compared to casein [10]. Some potential bone protective effects of SPI are the presence of estrogenic isoflavones and an amino acid profile associated with reduced acid load [11]. Diet acid load is a major determinant of calcium (Ca) excretion and in turn, bone mineral loss [11].

Different n-3 PUFA sources also affect bone. Growing female Sprague-Dawley rats fed fish oil rich in the longchain n-3 PUFAs, eicosapentaenoic acid (EPA, 20:5n-3) and docosahexaeonic acid (DHA, 22:6n-3), increased long bone BMD and BMC compared to rats fed corn oil low in n-3 PUFAs [12]. Growing female Hans:SPRD-cy rats, a non-orthologous model of human ADPKD, fed ALA-rich flaxseed oil had higher whole body BMC and BMD compared to animals fed corn oil [13]. To our knowledge no studies have investigated whether dietary n-3 PUFAs enhances bone health in ARPKD.

Studies on the effects of ARPKD on bone are lacking because treatment priority is on slowing disease progression to renal failure and early mortality [7]. Some drug therapies in development to treat polycystic kidney disease have reported side effects that include bone loss [14]. Also, many drugs used in the treatment of osteoporosis are not approved for use in patients with moderate to advanced kidney diseases [3]. Early diet management by optimizing bone health during the growth stage can potentially minimize bone loss due to renal dysfunction at an early age in polycystic kidney disease patients. Therefore, the objective of this study was to determine whether feeding growing female PCK rats SPI and n-3 PUFA can affect mineral balance and optimize bone health. Female rats were used because they are reportedly more efficient at metabolizing dietary ALA to the bioactive n-3 PUFAs, EPA and DHA [15]. Female Hans:SPRD-cy rats fed diets supplemented with phytoestrogens had more beneficial effects on PKD progression than male rats [16]. Also, slower progression of PKD in females enable the subtle influence of diet to be detectable [7].

\section{Methods}

\section{Animals and diets}

All animal procedures were conducted in accordance with the Institute of Laboratory Animal for the Care and Use of Laboratory Animals Guidelines [17], and were approved by the Animal Care and Use Committee at West Virginia University. Growing (age 28 d) female PCK rats were purchased from Charles River Laboratories (Wilmington,
MA). All rats were kept in a $21^{\circ} \mathrm{C}$ room with a 12 hour light/dark cycle throughout the 12 week study period. Following a 7- day acclimation period, animals were randomly assigned ( $\mathrm{n}=12$ rats/group) to experimental diets. A power analysis conducted prior to the experiment showed that with 10 rats/group at an alpha $=0.05$ there is $80 \%$ power to detect a difference in BMD and bone volume per unit of total volume (BV/TV) between the diet groups. An additional 2 rats/group was included due to possibility of morbidity or mortality due to disease severity in PCK rats.

All experimental diets were manufactured by Harlan Teklad (Madison, WI). Diet ingredients are provided in Table 1. The experimental diets were based on the American Institute of Nutrition-93G (AIN-93 G) standard rodent diet consisting of purified ingredients formulated to meet all the nutritional requirements of growing rats as defined by the National Research Council [18]. The AIN93G diet is formulated with casein as the protein source $(200 \mathrm{~g} / \mathrm{kg}$ diet $)$ and soybean oil as the lipid source $(70 \mathrm{~g} / \mathrm{kg}$ diet). In this study, experimental diets consisted of either casein or SPI as the protein source. SPI was generously provided by DuPont Nutrition and Health (St. Louis, $\mathrm{MO})$. Both protein sources consisted of $87 \%$ crude protein. Caloric value was $4.3 \mathrm{kcal} / \mathrm{g}$ for SPI and $4.2 \mathrm{kcal} / \mathrm{g}$ for casein.

The lipid sources consisted of corn oil because it is high in n-6 PUFA and low in n-3 PUFA which is typical of Western diets [19]. Soybean oil was included because it contains both essential fatty acids, the n- 6 PUFA, linoleic acid (LA, 18:2n-6) and the n-3 PUFA, ALA. A 1:1 ratio of soybean oil:salmon oil was included because it contains the n-3 PUFAs, ALA, EPA and DHA. Salmon oil was purchased from JEdwards International Inc (Quincy, MA). Caloric value was $9.2 \mathrm{kcal} / \mathrm{g}$ for corn oil, $9.1 \mathrm{kcal} / \mathrm{g}$ for soybean oil, and $9.3 \mathrm{kcal} / \mathrm{g}$ for the $1: 1$ soybean oil:salmon oil blend.

The experimental diets consisted of: 1) casein + corn oil $($ Casein $+\mathrm{CO}), 2)$ casein + soybean oil $($ Casein $+\mathrm{SO})$, 3) soy protein isolate + soybean oil $(\mathrm{SPI}+\mathrm{SO})$ or 4) soy protein isolate $+1: 1$ soybean/salmon oil $(\mathrm{SPI}+\mathrm{SB})$. Caloric value was $3.77 \mathrm{Kcal} / \mathrm{g}$ and metabolizable energy was $16.1 \pm 0.3 \mathrm{~kJ} / \mathrm{g}$ for all diets. Using inductively coupled plasma spectrometry (ICP, model P400, Perkin Elmer, Shelton, $\mathrm{CN}$ ), dietary $\mathrm{Ca}$ content was determined to be higher $(P=0.04)$ in the SPI $+\mathrm{SO}(9.7 \pm 0.1 \mathrm{mg} / \mathrm{g})$ and $\mathrm{SPI}+\mathrm{SB}(10.4 \pm 0.1 \mathrm{mg} / \mathrm{g})$ compared to Casein $+\mathrm{SO}$ $(8.8 \pm 0.1 \mathrm{mg} / \mathrm{g})$ and Casein $+\mathrm{CO}(8.2 \pm 0.2 \mathrm{mg} / \mathrm{g})$ diets. Dietary phosphorus $(\mathrm{P})$ content was determined to be higher $(P=0.03)$ in the SPI $+\mathrm{SO}(7.3 \pm 0.1 \mathrm{mg} / \mathrm{g})$ and $\mathrm{SPI}+\mathrm{SB}(7.2 \pm 0.1 \mathrm{mg} / \mathrm{g})$ compared to Casein $+\mathrm{SO}(6.1 \pm$ $0.5 \mathrm{mg} / \mathrm{g})$ and Casein $+\mathrm{CO}(6.5 \pm 0.2 \mathrm{mg} / \mathrm{g})$ diets.

All diets were kept at $-20^{\circ} \mathrm{C}$ until fed. Rats were provided their assigned diet and deonized distilled water $\left(\mathrm{ddH}_{2} \mathrm{O}\right)$ to prevent mineral intake from sources other 
Table 1 Experimental diet composition

\begin{tabular}{|c|c|c|c|c|}
\hline Ingredient (g/kg diet) $^{1}$ & Casein + CO & Casein + SO & $\mathrm{SPI}+\mathrm{SO}$ & $\mathrm{SPI}+\mathrm{SB}$ \\
\hline Casein & 200.0 & 200.0 & 0.0 & 0.0 \\
\hline Soy protein isolate & 0.0 & 0.0 & 200.0 & 200.0 \\
\hline L-Cystine & 3.0 & 3.0 & 1.3 & 1.3 \\
\hline Corn Starch & 397.5 & 397.5 & 397.5 & 397.5 \\
\hline Maltodextrin & 132.0 & 132.0 & 132.0 & 132.0 \\
\hline Sucrose & 100.0 & 100.0 & 100.0 & 100.0 \\
\hline Soybean Oil & 70.0 & 0.0 & 70.0 & 35.0 \\
\hline Corn Oil & 0.0 & 70.0 & 0.0 & 0.0 \\
\hline Salmon Oil & 0.0 & 0.0 & 0.0 & 35.0 \\
\hline Cellulose & 50.0 & 50.0 & 50.0 & 50.0 \\
\hline Mineral Mix ${ }^{2}$ & 35.0 & 35.0 & 35.0 & 35.0 \\
\hline Vitamin $\mathrm{Mix}^{2}$ & 10.0 & 10.0 & 10.0 & 10.0 \\
\hline Choline bitartrate & 2.5 & 2.5 & 2.5 & 2.5 \\
\hline TBHQ, antioxidant & 0.014 & 0.014 & 0.014 & 0.014 \\
\hline Calories & 377 & 377 & 377 & 377 \\
\hline \multicolumn{5}{|l|}{ Fatty acids (\% total fatty acids) } \\
\hline \multicolumn{5}{|l|}{$n-3$ PUFAs } \\
\hline Alpha-linolenic acid (ALA, 18:3n-3) & $0.59 \pm 0.18 c$ & $7.69 \pm 0.02^{\mathrm{a}}$ & $7.68 \pm 0.04^{\mathrm{a}}$ & $4.85 \pm 0.01^{b}$ \\
\hline Eicosapentaenoic acid (EPA, 20:5n-3) & ND & ND & ND & $7.4 \pm 0.03$ \\
\hline Docosahexaenoic acid (DHA, 22:6n-3) & ND & ND & ND & $3.9 \pm 0.1$ \\
\hline \multicolumn{5}{|l|}{$n-6$ PUFAs } \\
\hline Linoleic acid (LA, 18:2n-6) & $52.93 \pm 2.68^{\mathrm{a}}$ & $55.78 \pm 0.07^{\mathrm{a}}$ & $56.09 \pm 0.09^{\mathrm{a}}$ & $35.19 \pm 0.06^{b}$ \\
\hline Arachidonic acid (ARA, 20:4n-6) & $1.10 \pm 0.04^{\mathrm{a}}$ & $0.16 \pm 0.005^{c}$ & $0.21 \pm 0.01^{c}$ & $0.41 \pm 0.007^{b}$ \\
\hline$n-3 / n-6$ & 0.01 & 0.14 & 0.14 & 0.44 \\
\hline
\end{tabular}

${ }^{1}$ All ingredients are from Harlan Teklad except for SPI from DuPont Nutrition and Health (St. Louis, MO) and salmon oil from JEdwards International (Quincy, MA). ${ }^{2}$ Based on the AIN-93G vitamin and mineral mixes [36]. Different superscript letters a, b, c within the same rows indicate significant differences at $P<0.05$ by one-way ANOVA followed by Tukey's test. Abbreviations are CO corn oil, SO soybean oil, SPI soy protein isolate, SB 1:1 soybean oil:salmon oil blend, TBHQ tertiary butylhydroquinone, ND not detectable.

than the diet. Body weights were measured weekly throughout the 12 week study.

\section{Mineral balance}

To prevent variability in food intake, rats were provided $15 \pm 2 \mathrm{~g}$ of powdered diet daily. This amount has been reported to support growth based on body weights [20]. Food intake was measured daily and replaced with fresh diet. During the final week of the 12 weeks feeding study, rats were individually housed in metabolic cages to collect urine and feces. Final 7 day urine samples were collected, centrifuged at $1,500 \mathrm{~g}$ for $10 \mathrm{~min}$ at $4^{\circ} \mathrm{C}$, filtered through Whatman no. 1 paper, and diluted 1:10 in $\mathrm{ddH}_{2} \mathrm{O}$. Urinary $\mathrm{Ca}$ and $\mathrm{P}$ concentration were determined using ICP (model P400, Perkin Elmer, Shelton, CN).

Diet and fecal samples were freeze-dried for $48 \mathrm{~h}$, ashed in a muffle furnace at $550^{\circ} \mathrm{C}$ for $24 \mathrm{~h}$, and then acidified in $70 \%$ nitric acid. The acidified samples were neutralized in $\mathrm{ddH}_{2} \mathrm{O}$, filtered through Whatman no. 1 paper, diluted 1:50 in $\mathrm{dd}_{2} \mathrm{O}$, and both diet and fecal $\mathrm{Ca}$ and $\mathrm{P}$ content determined by ICP.

Following euthanasia by $\mathrm{CO}_{2}$ inhalation, the chest cavity was opened and the aorta punctured to collect blood. Blood was centrifuged at $1,500 \mathrm{~g}$ for $10 \mathrm{~min}$ at $4^{\circ} \mathrm{C}$ to obtain serum. Serum samples were stored at $-80^{\circ} \mathrm{C}$ until assayed. Serum parathyroid hormone $(\mathrm{PTH})$ was determined by commercially available rat enzyme immunoassay (EIA) kit (RayBiotech Inc., GA). Absorbance was determined at $450 \mathrm{~nm}$ using a Spectramax Plus microplate reader (Molecular Devices, Sunnyvale, CA). Serum Ca and $\mathrm{P}$ were determined enzymatically using a commercially available Vet-16 rotor and quantified by a Hemagen Analyst automated spectrophotometer (Hemagen Diagnostics Inc., Columbia, MD).

\section{Bone morphometry}

Following euthanization by $\mathrm{CO}_{2}$ asphyxiation, the right and left femurs and tibiae were collected ( $n=12$ rats/group). 
Bones were defleshed and morphometry measurements of medial lateral width, anterior posterior width, and length were determined using a vernier caliper (Bel-Art Products, Pequannock, NJ). Length was measured from the medial condyle to greater trochanter. Bones were dried at $110^{\circ} \mathrm{C}$ for $48 \mathrm{~h}$ then weighed using an analytical balance (Mettler Toledo, Columbus, OH). Morphometry measurements were averaged for bone pairs (i.e. right and left) after no bilateral differences were determined using paired $\mathrm{t}$-test with significance level set at $P<0.05$.

\section{Bone densitometry, Ash, $\mathrm{Ca}$, and $\mathrm{P}$}

Dual energy X-ray absorptiometry (DXA) scans (Hologic QDR 4500-A Elite) were performed on the left bones. Tibial and femoral specimens were placed in Millipore water. Bone mineral area (BMA), BMC, BMD were evaluated from all scans using the Regional High Resolution software package designed for studying isolated bone specimens (Hologic Waltham, MA).

Following DXA, bones were ashed in a muffle furnace (model CP18210, Thermolyne, Dubuque, IA) at $600^{\circ} \mathrm{C}$ for $24 \mathrm{~h}$ then weighed. Bone ash was dissolved in $2 \mathrm{~mL}$ of $70 \%$ nitric acid. Acidified samples were filtered through Whatman no. 1 paper and diluted $(1: 500 \mathrm{v} / \mathrm{v})$ to volume with $\mathrm{ddH}_{2} \mathrm{O}$. Bone $\mathrm{Ca}$ and $\mathrm{P}$ content were measured using ICP.

\section{Bone microarchitectural analyses}

Trabecular and cortical bone architecture was determined in the left bones using micro-computed tomography $(\mu \mathrm{CT})$ (MicroCT40, SCANCO Medical, Switzerland). Cortical bone architecture was evaluated on a $512 \mu \mathrm{m}$ region of the femur and tibia mid-diaphysis. Cortical indices included cortical thickness, cortical area, medullary area, and porosity. The trabecular bone within the distal femur metaphysis and proximal tibial metaphysis were scanned and $200 \mathrm{im}$ ages $(\sim 16 \mu \mathrm{m} /$ slice or $3.2 \mathrm{~mm})$ were analyzed with semiautomatically drawn contours beginning $400 \mu \mathrm{m}$ from the growth plate including only secondary spongiosa within the volume of interest. The volume of interest was assessed for structural parameters included trabecular BV/TV, trabecular number $(\mathrm{TbN})$, trabecular thickness $(\mathrm{TbTH})$, trabecular separation (TbSp), trabecular connectivity (Conn), and structure model index (SMI). All scans were performed utilizing a $1024 \times 1024$ matrix resulting in an isotropic voxel resolution of $22 \mu \mathrm{m}^{3}$. An integration time of 70 milliseconds per projection was used with a rotational step of $0.36^{\circ}$ resulting in total acquisition time of approximately $150 \mathrm{~min} / \mathrm{sample}$.

\section{Bone biomechanical strength}

Bone strength indices were assessed using a TA.XT2i Texture Analyzer (Texture Technologies, Scarsdale, NY) outfitted with a three-point bending apparatus. Femora and tibiae were placed on supports and force applied on the medial surface of the bones by lowering a centrally placed blade ( $1 \mathrm{~mm}$ width) at a constant crosshead speed $(0.1 \mathrm{~mm} / \mathrm{sec})$ until the bone was broken. The load cell was $250 \mathrm{~kg}$. The load-deflection data were collected by a PC interfaced with the TA.XT2i. Sample test distance was set at $10 \mathrm{~mm}$ with a signal collection rate of 100 points per sec. A force-displacement curve generated from the biomechanical test was used to determine bone strength measurements. Peak force is the highest load obtained before bone fracture occurs. Ultimate stiffness is the slope of the curve responding to bone stiffness [21]. Ultimate bending stress is a normalized calculated force that takes into account the size of the bone and Young's Modulus is a normalized calculated bone stiffness that takes into account bone size [22].

\section{Bone turnover markers}

Serum osteocalcin concentration was determined using a commercially available rat specific EIA (Biomedical Technologies, Stoughton, MA). Urinary deoxypyridinoline (DPD) concentration was determined by commercially available EIA kit (Quidel Corporation, CA). Serum osteocalcin was measured at $450 \mathrm{~nm}$ and urinary DPD was measured at $405 \mathrm{~nm}$ using a Spectramax Plus microplate reader.

\section{Statistical analysis}

Results are expressed as means \pm standard error of the mean (SEM). One way analysis of variance (ANOVA) was used to determine differences between treatment groups. Post-hoc multiple comparisons were performed using Tukey's test (parametric) or Wilcoxon rank test (non-parametric). Differences were considered significant at $P<0.05$. All statistical analyses were performed using SigmaStat 3.1 statistical software program (Systat Software Inc., San Jose, CA).

\section{Results}

\section{Body Weight and Bone Morphometry}

Growing female PCK rats fed different dietary treatments for 12 weeks had similar body weights (Figure 1). Femur length was shorter $(P=0.001)$ in PCK rats fed $\mathrm{SPI}+\mathrm{SO}$ diet compared to rats fed Casein $+\mathrm{CO}$ and Casein + SO diets (Table 2). There were no significant differences in femur width or dry weight between any of the diet groups. There were no differences in tibia length, width, and dry weight.

\section{Mineral intake and balance}

Urinary output was higher $(P=0.01)$ in rats fed SPI diets compared to rats fed casein diets, but there were no differences $(P=0.79)$ in water intake. Ca intake was significantly higher in rats fed SPI diets compared to rats fed 


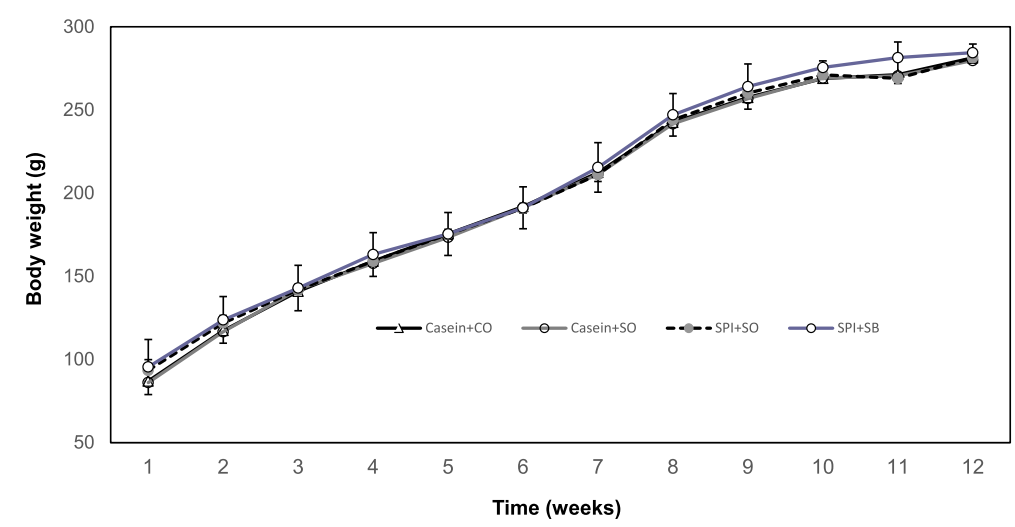

Figure 1 The effect of feeding growing PCK female rats soy protein isolate and/or omega-3 polyunsaturated fatty acids on body weight during the 12 week feeding study. Values are the mean \pm SEM of $n=12$ rats/diet group. Abbreviations are casein + corn oil (Casein + $\mathrm{CO}$ ), casein + soybean oil (Casein + SO), soy protein isolate + soybean oil (SPI + SO) or soy protein isolate + 1:1 soybean/salmon oil (SPI + SB).

casein diets. However, urinary Ca excretion was lower $(P=0.02)$ in rats fed SPI + SO diets compared to rats fed casein diets. Rats fed SPI diets had higher $(P=0.04) \mathrm{Ca}$ retention than rats fed casein diets. There was no significant differences in fecal Ca excretion or Ca apparent absorption between diet groups (Table 3).

$P$ intake was significantly higher in rats fed SPI diets compared to rats fed casein diets. However, urinary $\mathrm{P}$ excretion was lower $(P=0.01)$ in rats fed SPI diets compared to rats fed casein diets. Rats fed SPI diets had higher $(P=0.04) \mathrm{P}$ retention than rats fed casein diets. There were no significant differences in fecal $\mathrm{P}$ excretion or P apparent absorption between diet groups (Table 3 ). There were no significant differences in serum PTH, Ca or $\mathrm{P}$ concentrations among the dietary groups.

\section{Bone mineral analyses}

Shown in Table 4, there were no significant differences in femur or tibial $\mathrm{Ca}$ and $\mathrm{P}$ between diet groups. Additionally, there were no significant differences in long bone ash, BMA, BMC, and BMD between rats fed $\mathrm{Ca}-$ sein $+\mathrm{CO}$, Casein + SO, SPI + SO, and SPI + SB diets.

\section{Bone microarchitecture}

Shown in Table 5, there were significant differences in femur or tibia trabecular microarchitecture measurements of BV/TV, TbN, TbTH, TbSp, Conn or SMI in growing female $\mathrm{PCK}$ rats fed Casein $+\mathrm{CO}$, Casein $+\mathrm{SO}$, SPI $+\mathrm{SO}$, and SPI + SB diets.

There were also no significant differences in femur or tibia cortical thickness, cortical area, medullary area or porosity in growing female PCK rats fed different diets (Table 6).

\section{Bone biomechanical strength}

There was no significant difference in femur or tibia biomechanical strength measures of peak force, ultimate stiffness, ultimate bending stress, and Young's Modulus between the different diet groups (Table 7).

Table 2 Bone morphometry in growing female PCK rats fed different diets

\begin{tabular}{|c|c|c|c|c|c|}
\hline Measurement* & Casein + CO & Casein + SO & $\mathrm{SPI}+\mathrm{SO}$ & $\mathrm{SPI}+\mathrm{SB}$ & $P$-value \\
\hline \multicolumn{6}{|l|}{ Femur } \\
\hline Length (mm) & $31.01 \pm 0.13^{a}$ & $31.23 \pm 0.15^{\mathrm{a}}$ & $30.17 \pm 0.28^{b}$ & $30.74 \pm 0.14^{\mathrm{ab}}$ & 0.001 \\
\hline Medial Lateral width (mm) & $3.77 \pm 0.02$ & $3.75 \pm 0.03$ & $3.78 \pm 0.02$ & $3.79 \pm 0.03$ & 0.78 \\
\hline Anterior posterior width (mm) & $2.78 \pm 0.03$ & $2.77 \pm 0.03$ & $2.75 \pm 0.03$ & $2.84 \pm 0.03$ & 0.22 \\
\hline Dry weight (g) & $0.69 \pm 0.04$ & $0.70 \pm 0.04$ & $0.68 \pm 0.46$ & $0.68 \pm 0.04$ & 0.98 \\
\hline \multicolumn{6}{|l|}{ Tibia } \\
\hline Length (mm) & $36.24 \pm 0.15$ & $36.45 \pm 0.07$ & $36.20 \pm 0.07$ & $36.17 \pm 0.11$ & 0.25 \\
\hline Medial Lateral width (mm) & $2.24 \pm 0.02$ & $2.29 \pm 0.02$ & $2.24 \pm 0.02$ & $2.29 \pm 0.02$ & 0.18 \\
\hline Anterior posterior width (mm) & $2.68 \pm 0.02$ & $2.67 \pm 0.02$ & $2.67 \pm 0.02$ & $2.66 \pm 0.02$ & 0.88 \\
\hline Dry weight (g) & $0.47 \pm 0.02$ & $0.48 \pm 0.02$ & $0.46 \pm 0.02$ & $0.47 \pm 0.02$ & 0.95 \\
\hline
\end{tabular}

*Values are the means \pm SEM of $n=12$ rats/group. Different superscript letters $\mathrm{a}, \mathrm{b}$ within the same rows indicate significant differences at $P<0.05$ by one-way ANOVA followed by Tukey's test. Abbreviations are CO corn oil, SO soybean oil, SPI soy protein isolate, SB 1:1 soybean oil:salmon oil blend. 
Table 3 Calcium and phosphorus balance in growing female PCK rats fed different diets

\begin{tabular}{|c|c|c|c|c|c|}
\hline Measurement* & Casein + CO & Casein + SO & $\mathrm{SPI}+\mathrm{SO}$ & $\mathrm{SPI}+\mathrm{SB}$ & $P$-value \\
\hline Water intake $(\mathrm{mL} / \mathrm{d})$ & $14.4 \pm 9.4$ & $15.2 \pm 13.7$ & $16.3 \pm 11.5$ & $16.5 \pm 12.3$ & 0.79 \\
\hline Urinary Output (mL/d) & $2.1 \pm 0.2 b$ & $2.3 \pm 0.2 b$ & $3.8 \pm 0.3 a$ & $3.5 \pm 0.3 a$ & 0.01 \\
\hline Fecal Output (mg/d) & $5.2 \pm 0.3$ & $5.0 \pm 0.3$ & $5.5 \pm 0.3$ & $5.7 \pm 0.2$ & 0.21 \\
\hline \multicolumn{6}{|l|}{ Calcium } \\
\hline Ca Intake (mg/d) & $131.3 \pm 2.4 b$ & $123.0 \pm 2.2 b$ & $144.7 \pm 2.1 \mathrm{a}$ & $156.6 \pm 2.4 a$ & $<0.001$ \\
\hline Urinary Ca Excretion $(\mathrm{mg} / \mathrm{mL})^{1}$ & $1.9 \pm 0.3 a$ & $1.6 \pm 0.5 a$ & $0.5 \pm 0.07 b$ & $1.3 \pm 0.3 a b$ & 0.02 \\
\hline Fecal Ca Excretion (mg/d) & $29.9 \pm 2.3$ & $27.2 \pm 2.3$ & $26.0 \pm 2.2$ & $29.0 \pm 2.7$ & 0.65 \\
\hline Ca Apparent Absorption (\%) ${ }^{2}$ & $77.2 \pm 1.7$ & $77.9 \pm 1.9$ & $82.0 \pm 1.5$ & $81.5 \pm 1.7$ & 0.12 \\
\hline Ca Retention $(\mathrm{mg} / \mathrm{d})^{3}$ & $75.7 \pm 1.7 b$ & $76.6 \pm 1.8 b$ & $81.7 \pm 1.5 a$ & $80.6 \pm 1.7 a$ & 0.04 \\
\hline \multicolumn{6}{|l|}{ Phosphorus } \\
\hline P Intake (g) & $91.8 \pm 1.0 b$ & $97.0 \pm 2.1 b$ & $109.8 \pm 2.0 a$ & $108.6 \pm 2.3 a$ & $<0.001$ \\
\hline Urinary P Excretion $(\mathrm{mg} / \mathrm{mL})^{4}$ & $7.9 \pm 1.0 a$ & $6.8 \pm 1.3 a$ & $3.4 \pm 0.6 b$ & $2.3 \pm 0.6 b$ & 0.01 \\
\hline Fecal P Excretion (mg/d) & $26.1 \pm 2.6$ & $28.4 \pm 1.3$ & $28.3 \pm 2.5$ & $26.3 \pm 2.7$ & 0.85 \\
\hline P Apparent Absorption (\%) & $71.6 \pm 2.9$ & $70.7 \pm 1.4$ & $74.2 \pm 2.3$ & $75.7 \pm 2.5$ & 0.42 \\
\hline P Retention $(\mathrm{mg} / \mathrm{d})^{6}$ & $63.0 \pm 3.1 b$ & $63.7 \pm 1.8 b$ & $71.1 \pm 2.5 a$ & $73.6 \pm 2.3 a$ & 0.04 \\
\hline Serum parathyroid hormone $(\mathrm{pg} / \mathrm{mL})$ & $36.2 \pm 4.1$ & $37.2 \pm 4.4$ & $39.9 \pm 5.2$ & $43.6 \pm 5.9$ & 0.74 \\
\hline Serum Ca $(\mathrm{mg} / \mathrm{mL})$ & $0.12 \pm 0.004$ & $0.12 \pm 0.001$ & $0.11 \pm 0.004$ & $0.11 \pm 0.004$ & 0.17 \\
\hline Serum P (mg/mL) & $0.12 \pm 0.006$ & $0.13 \pm 0.007$ & $0.11 \pm 0.006$ & $0.12 \pm 0.005$ & 0.32 \\
\hline
\end{tabular}

"Values are expressed as the mean ${ }_{ \pm}$SEM of $\mathrm{n}=12$ rats/group. Different letters $\mathrm{a}, \mathrm{b}$ within the same column indicate significant differences at $P<0.05$ by one-way ANOVA followed by Tukey's test. Abbreviations are SO soybean oil, SPI soy protein isolate, SB 1:1 soybean oil:salmon oil blend, Ca calcium; $P$ phosphorus.

${ }^{1}$ Urinary calcium excretion was calculated as urinary Ca concentration/urine volume.

${ }^{2}$ Calcium apparent absorption was calculated as [(Ca intake - fecal Ca excretion)/Ca intake] x 100.

${ }^{3}$ Calcium retention was determined by calculating [Ca intake - (fecal Ca excretion + urinary Ca excretion)].

${ }^{4}$ Urinary phosphorus excretion was calculated as urinary $\mathrm{P}$ concentration/urine volume.

${ }^{5}$ Phosphorus apparent absorption was calculated as $[(\mathrm{P}$ intake - fecal $\mathrm{P}$ excretion $) / \mathrm{P}$ intake $] \times 100$.

${ }^{6}$ Phosphours retention was determined by calculating [P intake - (fecal P excretion + urinary $\mathrm{P}$ excretion)].

Table 4 Bone mineralization in growing female PCK rats fed different diets

\begin{tabular}{|c|c|c|c|c|c|}
\hline Measurement* & Casein + CO & Casein + SO & $\mathrm{SPI}+\mathrm{SO}$ & $\mathrm{SPI}+\mathrm{SB}$ & $P$-value \\
\hline \multicolumn{6}{|l|}{ Femur } \\
\hline $\mathrm{Ca}$ (mg/g of bone) & $93.0 \pm 1.0$ & $90.7 \pm 1.7$ & $94.3 \pm 0.7$ & $94.0 \pm 1.5$ & 0.23 \\
\hline $\mathrm{P}$ (mg/g of bone) & $50.1 \pm 0.5$ & $49.0 \pm 0.9$ & $50.5 \pm 0.4$ & $50.5 \pm 0.7$ & 0.39 \\
\hline Ash (mg/g bone) & $612.0 \pm 7.3$ & $578.9 \pm 9.6$ & $601.3 \pm 8.2$ & $614.5 \pm 8.4$ & 0.66 \\
\hline $\mathrm{BMA}\left(\mathrm{cm}^{2}\right)$ & $1.30 \pm 0.01$ & $1.29 \pm 0.02$ & $1.28 \pm 0.01$ & $1.30 \pm 0.01$ & 0.27 \\
\hline BMC (g) & $0.23 \pm 0.0001$ & $0.23 \pm 0.0002$ & $0.22 \pm 0.0001$ & $0.23 \pm 0.0001$ & 0.67 \\
\hline $\mathrm{BMD}\left(\mathrm{mg} / \mathrm{cm}^{2}\right)$ & $0.18 \pm 0.0001$ & $0.18 \pm 0.0002$ & $0.17 \pm 0.0001$ & $0.18 \pm 0.0002$ & 0.35 \\
\hline \multicolumn{6}{|l|}{ Tibia } \\
\hline $\mathrm{Ca}$ (mg/g of bone) & $90.7 \pm 1.2$ & $93.0 \pm 1.1$ & $96.8 \pm 2.5$ & $95.5 \pm 1.9$ & 0.14 \\
\hline P (mg/g of bone) & $46.8 \pm 0.9$ & $48.3 \pm 0.6$ & $49.9 \pm 1.3$ & $49.5 \pm 1.0$ & 0.12 \\
\hline Ash (mg/g bone) & $602.5 \pm 5.9$ & $592.1 \pm 7.8$ & $603.0 \pm 7.5$ & $606.7 \pm 4.1$ & 0.59 \\
\hline $\mathrm{BMA}\left(\mathrm{cm}^{2}\right)$ & $1.54 \pm 0.04$ & $1.50 \pm 0.06$ & $1.56 \pm 0.06$ & $1.49 \pm 0.04$ & 0.56 \\
\hline BMC (g) & $0.33 \pm 0.01$ & $0.32 \pm 0.01$ & $0.33 \pm 0.01$ & $0.31 \pm 0.02$ & 0.48 \\
\hline $\mathrm{BMD}\left(\mathrm{mg} / \mathrm{cm}^{2}\right)$ & $0.21 \pm 0.002$ & $0.21 \pm 0.003$ & $0.21 \pm 0.001$ & $0.21 \pm 0.002$ & 0.68 \\
\hline
\end{tabular}

*Values are the means \pm SEM of $\mathrm{n}=12$ rats/group. Abbreviations are CO corn oil, SO soybean oil, SPI soy protein isolate, SB 1:1 soybean oil:salmon oil blend, $B M A$ bone mineral area, $B M C$ bone mineral content, $B M D$ bone mineral density, $C a$ calcium, $P$ phosphorus. 
Table 5 Trabecular bone microarchitecture in growing female PCK rats fed different diets

\begin{tabular}{|c|c|c|c|c|c|}
\hline Measurement ${ }^{*}$ & Casein + CO & Casein + SO & $\mathrm{SPI}+\mathrm{SO}$ & $\mathrm{SPI}+\mathrm{SB}$ & $P$-value \\
\hline \multicolumn{6}{|l|}{ Femur } \\
\hline BV/TV (\%) & $14.30 \pm 0.54$ & $14.00 \pm 0.58$ & $12.95 \pm 0.96$ & $13.64 \pm 0.86$ & 0.61 \\
\hline TbN (per mm) & $2.87 \pm 0.11$ & $2.74 \pm 0.11$ & $2.77 \pm 0.20$ & $2.86 \pm 0.10$ & 0.87 \\
\hline TbTh (mm) & $0.07 \pm 0.0009$ & $0.07 \pm 0.001$ & $0.06 \pm 0.001$ & $0.07 \pm 0.002$ & 0.18 \\
\hline TbSp (mm) & $0.36 \pm 0.01$ & $0.38 \pm 0.01$ & $0.40 \pm 0.04$ & $0.36 \pm 0.01$ & 0.65 \\
\hline Conn $\left(1 / \mathrm{mm}^{3}\right)$ & $80.77 \pm 2.99$ & $76.47 \pm 2.83$ & $77.06 \pm 5.26$ & $77.83 \pm 3.59$ & 0.86 \\
\hline SMI & $2.28 \pm 0.03$ & $2.30 \pm 0.05$ & $2.12 \pm 0.06$ & $2.36 \pm 0.07$ & 0.31 \\
\hline \multicolumn{6}{|l|}{ Tibia } \\
\hline BV/TV (\%) & $9.43 \pm 0.62$ & $9.67 \pm 0.63$ & $9.13 \pm 0.84$ & $9.24 \pm 0.83$ & 0.96 \\
\hline TbN (per mm) & $2.51 \pm 0.13$ & $2.44 \pm 0.17$ & $2.39 \pm 0.19$ & $2.61 \pm 0.16$ & 0.82 \\
\hline TbTh (mm) & $0.06 \pm 0.001$ & $0.06 \pm 0.0009$ & $0.06 \pm 0.001$ & $0.06 \pm 0.001$ & 0.33 \\
\hline TbSp (mm) & $0.42 \pm 0.02$ & $0.44 \pm 0.30$ & $0.46 \pm 0.05$ & $0.41 \pm 0.03$ & 0.63 \\
\hline Conn $\left(1 / \mathrm{mm}^{3}\right)$ & $43.00 \pm 4.56$ & $43.58 \pm 4.59$ & $43.53 \pm 6.11$ & $43.26 \pm 6.34$ & 1.00 \\
\hline SMI & $2.85 \pm 0.05$ & $2.80 \pm 0.04$ & $2.88 \pm 0.07$ & $2.88 \pm 0.07$ & 0.72 \\
\hline
\end{tabular}

\section{Bone turnover markers}

There were no significant differences in serum osteocalcin (Figure 2A) and urinary DPD concentration (Figure 2B) in growing female PCK rats fed the different experimental diets.

\section{Discussion}

Hyperphosphatemia, hypocalcemia and hyperparathyroidism in children with chronic renal failure plays a significant role in bone disorders [1]. Liver disease can also contribute to bone disorders through impaired vitamin $\mathrm{D}$ synthesis which results in decreased $\mathrm{Ca}$ absorption and a compensatory increase in PTH production [23]. Despite greater $(P=0.01)$ urine output in the absence of higher water intake, urinary $\mathrm{Ca}$ excretion was significantly lower in rats fed SPI + SO diet compared to rats fed casein diets. Similarly, urinary $P$ excretion was lower $(P=0.01)$ in SPI than casein-fed rats. Higher dietary $\mathrm{Ca}$ and $\mathrm{P}$ content in SPI diets resulted in greater $\mathrm{Ca}$ and $\mathrm{P}$ consumption. Lower urinary excretion and higher intake resulted in greater $(P=0.04) \mathrm{Ca}$ and $\mathrm{P}$ retention in SPI-fed rats. PTH decreases $\mathrm{Ca}$ and $\mathrm{P}$ excretion in the urine; however, in the current study there were no statistical differences in serum PTH concentration among the diet groups. Failing kidneys lose capacity to concentrate urine. Plischke et al. [24] showed evidence linking fluid intake, vasopression, and osmotic control in PKD progression. In our study, reduced urinary $\mathrm{Ca}$ and $\mathrm{P}$ excretion in rats fed SPI diet may be due to lower urinary osmolality. Growing female PCK rats fed $\mathrm{SPI}+\mathrm{SO}$ and $\mathrm{SPI}+\mathrm{SB}$ diet had impaired renal

Table 6 Cortical bone microarchitecture in growing female PCK rats fed different diets

\begin{tabular}{|c|c|c|c|c|c|}
\hline Measurement* & Casein + CO & Casein + SO & $\mathrm{SPI}+\mathrm{SO}$ & $\mathrm{SPI}+\mathrm{SB}$ & $P$ value \\
\hline \multicolumn{6}{|l|}{ Femur } \\
\hline Cortical Thickness (mm) & $0.55 \pm 0.008$ & $0.55 \pm 0.004$ & $0.56 \pm 0.004$ & $0.55 \pm 0.004$ & 0.47 \\
\hline Cortical Area $\left(\mathrm{mm}^{2}\right)$ & $4.49 \pm 0.03$ & $4.40 \pm 0.05$ & $4.56 \pm 0.03$ & $4.52 \pm 0.05$ & 0.06 \\
\hline Medullary Area $\left(\mathrm{mm}^{2}\right)$ & $0.26 \pm 0.006$ & $0.25 \pm 0.007$ & $0.25 \pm 0.006$ & $0.26 \pm 0.007$ & 0.94 \\
\hline Porosity (\%) & $5.42 \pm 0.12$ & $5.45 \pm 0.15$ & $5.28 \pm 0.14$ & $5.39 \pm 0.12$ & 0.78 \\
\hline \multicolumn{6}{|l|}{ Tibia } \\
\hline Cortical Thickness (mm) & $0.52 \pm 0.004$ & $0.53 \pm 0.003$ & $0.53 \pm 0.01$ & $0.53 \pm 0.006$ & 0.59 \\
\hline Cortical Area $\left(\mathrm{mm}^{2}\right)$ & $2.90 \pm 0.03$ & $2.94 \pm 0.03$ & $2.88 \pm 0.05$ & $2.98 \pm 0.04$ & 0.29 \\
\hline Medullary Area $\left(\mathrm{mm}^{2}\right)$ & $0.20 \pm 0.004$ & $0.19 \pm 0.003$ & $0.18 \pm 0.004$ & $0.19 \pm 0.005$ & 0.46 \\
\hline Porosity (\%) & $6.32 \pm 0.02$ & $6.06 \pm 0.09$ & $6.11 \pm 0.13$ & $6.04 \pm 0.16$ & 0.37 \\
\hline
\end{tabular}

*Values are expressed as the mean \pm SEM of $n=12$ bones/group. Abbreviations are CO corn oil, SO soybean oil, SPI soy protein isolate, SB 1:1 soybean oil:salmon oil. 
Table 7 Bone strength measurements in growing female PCK rats fed different diets

\begin{tabular}{|c|c|c|c|c|c|}
\hline Measurement* & Casein + CO & Casein + SO & $\mathrm{SPI}+\mathrm{SO}$ & $\mathrm{SPI}+\mathrm{SB}$ & $P$ value \\
\hline \multicolumn{6}{|l|}{ Femur } \\
\hline Peak Force (N) & $37.2 \pm 2.6$ & $42.5 \pm 2.7$ & $40.4 \pm 2.6$ & $44.5 \pm 3.0$ & 0.26 \\
\hline Ultimate Stiffness (N/S) & $437.6 \pm 23.3$ & $437.7 \pm 37.1$ & $385.6 \pm 33.7$ & $429.4 \pm 29.8$ & 0.63 \\
\hline Ultimate Bending Stress $\left(\mathrm{N} / \mathrm{mm}^{2}\right)$ & $41.8 \pm 3.2$ & $47.3 \pm 2.3$ & $45.7 \pm 3.0$ & $48.6 \pm 3.5$ & 0.40 \\
\hline Young's Modulus (N/mm²) & $1160.6 \pm 54.0$ & $1171.5 \pm 106.2$ & $1040.0 \pm 100.6$ & $1117.8 \pm 99.2$ & 0.75 \\
\hline \multicolumn{6}{|l|}{ Tibia } \\
\hline Peak Force $(\mathrm{N})$ & $19.2 \pm 1.3$ & $22.3 \pm 1.7$ & $20.0 \pm 1.7$ & $20.7 \pm 1.3$ & 0.57 \\
\hline Ultimate Stiffness (N/S) & $178.0 \pm 13.1$ & $171.3 \pm 13.0$ & $158.9 \pm 7.9$ & $161.2 \pm 10.9$ & 0.61 \\
\hline Ultimate Bending Stress $\left(\mathrm{N} / \mathrm{mm}^{2}\right)$ & $51.5 \pm 3.4$ & $61.7 \pm 4.0$ & $56.37 \pm 5.58$ & $57.41 \pm 5.26$ & 0.5 \\
\hline Young's Modulus (N/mm²) & $1938.8 \pm 166.7$ & $1970.0 \pm 172.8$ & $1815.6 \pm 125.1$ & $1765.5 \pm 150.5$ & 0.76 \\
\hline
\end{tabular}

*Values are expressed as the mean \pm SEM of $n=12$ bone pairs/group. Abbreviations are CO corn oil, SO soybean oil, SPI soy prtein isolate, SB 1:1 soybean oil: salmon oil blend.

function indicated by elevated serum blood urea nitrogen concentration compared to casein-fed rats [25]. Rats fed SPI + SB diet had significantly more renal cortical cyst obstruction [25] as well as significantly higher serum alkaline phosphatase and bilirubin concentrations due to hepatic cyst obstruction of the bile duct [26].
Despite higher $\mathrm{Ca}$ and $\mathrm{P}$ retention there were no differences on long bone $\mathrm{Ca}$ and $\mathrm{P}$ content in SPI compared to casein-fed rats. There were also no differences in femur or tibial BMA, BMC, and BMD as determined by DXA. In contrast, feeding growing female SpragueDawley rats SPI for 14 days enhanced tibial BMC and

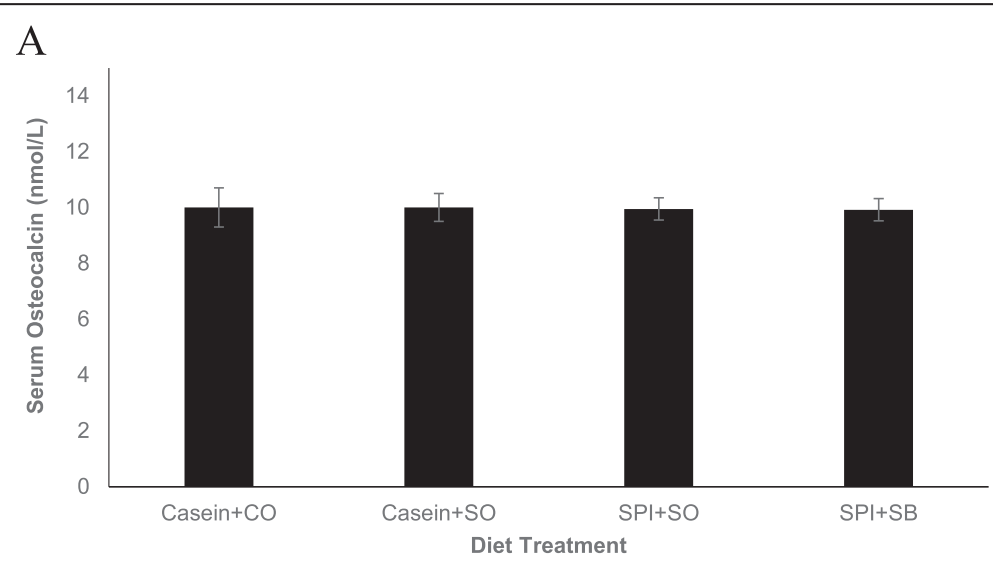

B

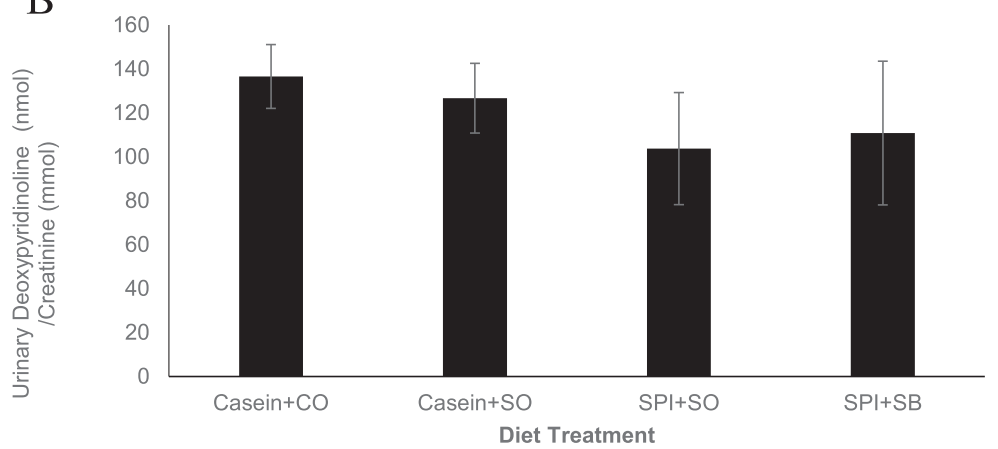

Figure 2 The effect of feeding growing PCK female rats on soy protein isolate and/or omega-3 polyunsaturated fatty acids on body weight for 12 weeks on A) serum osteocalcin concentration and B) urinary deoxypyridinoline concentration. Bars represent the mean \pm $\mathrm{SEM}$ of $\mathrm{n}=10$ rats/diet group. Abbreviations are casein + corn oil (Casein $+\mathrm{CO})$, casein + soybean oil (Casein + SO), soy protein isolate + soybean oil $(\mathrm{SPI}+\mathrm{SO})$ or soy protein isolate + 1:1 soybean/salmon oil (SPI + SB). 
BMD compared to casein [10]. Differences may be due to a disease versus non-disease state. Also, metabolic abnormalities accompanying renal disease effects on bone microarchitecture cannot be detected by DXA [27]. Using $\mu \mathrm{CT}$ technology, we found no significant differences in femur and tibia trabecular microarchitecture in growing female PCK rats fed different diets. Lukas et al. [12] reported that growing female Sprague-Dawley rats fed oil sources rich in ALA increased femur and tibia BV/ $\mathrm{TV}, \mathrm{TbN}, \mathrm{TbTH}$ and reduced TbSp by promoting bone formation indicated by higher serum osteocalcin concentration and no significant difference in bone resorption. In the present study, growing female PCK rats fed soybean oil or soybean oil: fish oil blend showed no significant differences in serum osteocalcin or urinary DPD concentration compared to rats fed corn oil. In our study, soybean oil was provided as a source of ALA, whereas in the Lukas et al. [12] study, flaxseed oil was used. The ALA content of flaxseed oil is higher 57\% [20] than soybean oil (7.69\%) or soybean:salmon oil blend (4.85\%). Also, a higher dose $(12 \% \mathrm{wt})$ of pure flaxseed oil or fish oil was provided to Sprague-Dawley rats compared to the oil dose (7\% wt) provided to PCK rats. Therefore, higher n-3 PUFA doses may be necessary to enhance trabecular bone microarchitecture in growing female PCK rats. High fat diets were not fed to PCK rats due to potential to worsen polycystic kidney disease progression and severity [28]. Also, hypertension and cardiovascular defects are frequent complications of PKD that need to be considered when recommending a high fat diet [29-31]. In children with chronic kidney disease, cardiovascular disease is the most common cause of death and this has been suggested to be due in part to dysregulation of $\mathrm{Ca}$ and $\mathrm{P}$ homeostasis causing vascular calcification [32]. We did not assess vascular calcification, but found no significant differences in serum PTH, Ca or P concentration between diet groups.

In kidney disease, elevated PTH has been reported to preferentially cause cortical bone loss [27,33]. In the present study, no significant differences in femur and tibia cortical bone microarchitecture were observed in growing female PCK fed diets containing different protein and lipid sources. Growing female Sprague-Dawley rats fed oils rich in ALA, EPA, and/or DHA also showed no effect on cortical bone microarchitecture [12]. As expected in the absence of significant differences in bone mass and microarchitecture there were no significant differences in bone strength measurements between growing female PCK rats fed different diets.

Besides mineral imbalance and failure to achieve PBM, children with kidney disease also suffer growth disturbances [34]. Early management is required to prevent growth failure and shorter stature [2]. In the present study, all diets supported growth indicated by no significant differences in body weights. A long-term (two years) study showed no difference in body weight gain in Sprague-Dawley rats fed a soy protein compared to a casein-based diet [35]. Similar body weight gain has also been reported for growing rats fed soybean oil, flaxseed oil, and fish oil $[20,36]$. Hans: SPRD-cy rats provided soy protein ad libitum had higher body weights and showed improved kidney cyst volume and renal function compared to rats fed casein [37]. In our study female PCK rats fed SPI diets showed higher final urinary output and decreased renal function compared to casein-fed rats; however, there were no significant body weight differences. This may have been because all diets were restricted to $15 \mathrm{~g} / \mathrm{d}$.

In our study, femur length was shorter in rats fed SPI + SO diet compared to rats fed casein diets. SPI contains isoflavone phytoestrogens with binding specificity for estrogen receptor beta [38]. Estrogen receptor beta has been shown to be a physiological inhibitor of bone growth [39]. Tou et al. [40] reported that female Sprague-Dawley rats fed a phytoestrogen diet shortened $(P<0.05)$ femur length compared to rats fed a casein diet. However, PCK rats fed SPI + SB diet had no effect on femur length. The type of lipid associated with SPI may influence bone. Of the diet groups, SPI + SB diet consisting of a soybean oil:fish oil blend had the highest n-3:n-6 PUFA ratio as well as EPA and DHA content. Growing female Hans:SPRD-cy rats, a model of ADPKD, fed casein with 7\% wt ALA-rich flaxseed oil for 12 weeks resulted in longer femurs compared to rats fed corn oil low in ALA [41]. ALA can be converted to EPA and DHA de novo [42]. Although conversion is inefficient, higher amounts of ALA associated with flaxseed oil can result in higher EPA and DHA content. Mechanisms whereby n-3 PUFAs have been suggested to promote bone growth include reducing prostaglandin $E_{2}$ production by decreasing the $n-6$ to $n-3$ PUFA ratio and by increasing circulating insulin-like growth factor binding protein to promote growth factors [43,44]. Estrogen can simulate the conversion of ALA into their longer-chain metabolites [45]. Therefore, a potential synergistic effect of SPI and n-3 PUFAs is estrogenic effects of isoflavones associated with SPI may increase conversion of ALA to long-chain n-3 PUFAs. Female PCK rats fed soybean oil with protein source provided as either SPI or casein showed no significant differences in serum and liver EPA, DHA, and ARA content $[25,26]$. However, dietary groups in our study did not allow us to specifically assess synergistic effects of protein and n-3 PUFAs on femur longitudinal growth.

Another limitation of this study was the AIN-93G diet is based on a purified casein diet. This standard purified diet does not meet the NRC recommendations for sulfur amino acids to support rodent growth and requires the addition of L-cysteine [46]. Therefore, SPI diets were supplemented with L-cysteine to equal casein diets. This 
potentially alters acid load typically associated with the amino acid profiles of these proteins. Also, a longer study duration than 12 weeks may be required to detect dietary effects of enhance bone mineralization due to higher $\mathrm{Ca}$ and $\mathrm{P}$ retention. However, ARPKD onset occurs early in childhood with renal failure frequently occurring in the first decade of life [6]. Although, the PCK rat model has the advantage of being a slow progressing form of ARPKD, the study was ended at 12 weeks due to morbidity associated with severity of polycystic liver [26,47].

\section{Conclusion}

In summary, to our knowledge this was the first study to investigate diet as a therapeutic strategy aimed at bone health in PCK rats, an orthologous model of human ARPKD $[8,47]$. Current general dietary guidelines to promote a healthier population include increasing consumption of plant proteins and n-3 PUFAs [48]. Growing female PCK rats fed SPI diets increased Ca and $\mathrm{P}$ retention, but not bone $\mathrm{Ca}$ and $\mathrm{P}$ content. All diets equally supported body weight gain and produced no greater or worse long bone mass, microarchitecture, and strength, but femur longitudinal growth was affected. Based on the results dietary SPI and n-3 PUFA supplementation influenced mineral balance without enhancing bone health in ARPKD.

\begin{abstract}
Abbreviations
ADPKD: Autosomal recessive polycystic kidney disease; AIN-93G: American Institute of Nutrition-93G; ALA: Alpha-linolenic acid; ARPKD: Autosomal recessive polycystic kidney disease; BMA: Bone mineral area; BMC: Bone mineral content; BMD: Bone mineral density; BV/TV: Bone volume per unit of total volume; $\mathrm{Ca}$ : Calcium; $\mathrm{ddH}_{2} \mathrm{O}$ : Deionized distilled water; DPD: Deoxypyridinoline; DHA: Docosahexaeonic acid; DXA: Dual energy X-ray absorptiometry; ElA: Enzyme immunoassay; EPA: Eicosapentaenoic acid; ICP: Inductively coupled plasma spectrometry; LA: Linoleic acid; n-3 PUFA: Omega-3 polyunsaturated fatty acids; n-6 PUFA: Omega-6 polyunsaturated fatty acids; $\mu \mathrm{CT}$ : Micro-computed tomography; PBM: Peak bone mass; P: Phosphorus; SB: Soybean oil:salmon oil blend; SEM: Standard error of the mean; SPI: Soy protein isolate; SMI: Structure model index; SO: Soybean oil; TbN: Trabecular number; TbTH: Trabecular thickness; TbSp: Trabecular separation.
\end{abstract}

\section{Competing interests}

The authors declare that they have no competing interests.

\section{Authors' contributions}

KM and JCT designed the study, performed research, analyzed data, and wrote the manuscript. BJS performed the bone DXA and $\mu \mathrm{CT}$, analyzed data, interpreted data, and reviewed the manuscript. MM performed research, analyzed data, interpreted data, and reviewed the manuscript. CO performed research and reviewed the manuscript. All authors read and approved the final manuscript.

\section{Acknowledgements}

We thank Dr. Lewis MacDonald for use of the ICP, Joan Wright for performing the ICP analysis, Dr. Barbara Mickelson for her expertise in formulating and manufacturing the diet, and DuPont Nutrition and Health for generously donating the SPI to our laboratory. This manuscript is published with the approval of the director of the West Virginia Agricultural and Forestry Experimental Station as scientific paper number 3231.

\section{Funding}

This project was supported by the United Soybean Board Soybean Health Incentive Grant, West Virginia University PSCoR 10008736.6-11-NT10055R, West Virginia University Agriculture and Forestry Experimental Station Hatch Grant WAA00665, and West Virginia University summer undergraduate education program.

\section{Author details}

${ }^{1}$ Division of Animal and Nutritional Sciences, West Virginia University, 1038 Agricultural Sciences Bldg, P.O. Box 6108, Evansdale Campus, Morgantown, West Virginia 26505, USA. ²Department of Nutritional Sciences, Oklahoma State University, Stillwater, OK 74078, USA.

Received: 16 June 2014 Accepted: 23 January 2015

Published online: 10 February 2015

\section{References}

1. Wesseling-Perry K, Salusky IB. Chronic kidney disease: mineral and bone disorder in children. Semin Nephrol. 2013;33:169-79.

2. Bacchetta J, Harambat J, Cochat P, Salusky IB, Wesseling-Perry K. The consequences of chronic kidney disease on bone metabolism and growth in children. Nephrol Dial Transplant. 2012;27:3063-71.

3. Stompor T, Zabłocki M, Łesiów M. Osteoporosis in mineral and bone disorders of chronic kidney disease. Pol Arch Med Wewn. 2013;123:314-20.

4. Torres VE, Harris PC. Mechanisms of disease: autosomal dominant and recessive polycystic kidney diseases. Nat Clin Pract Nephrol. 2006;2:40-55.

5. Benun J, Lewis C. Polycystic kidney disease. Pediatr Rev. 2009;30:e78-9.

6. Dell KMR, Avner ED. Polycystic kidney disease: autosomal recessive. In: Pagon RA, Adam MP, Bird TD, Dolan CR, Fong CT, Stephens K, editors. GeneReviews $^{\text {TM }}$ [Internet]. Seattle (WA): University of Washington, Seattle; 2012. p. 1993-2013.

7. Maditz K, Gigliotti JC, Tou JC. Evidence for a role of proteins, lipids, and phytochemicals in the prevention of polycystic kidney disease progression and severity. Nutr Rev. 2013;7:802-14.

8. Blazer-Yost BL, Haydon J, Eggleston-Gulyas T, Chen JH, Wang X, Gattone V, et al. Pioglitazone attenuates cystic burden in the PCK rodent model of polycystic kidney disease. PPAR Res. 2010;274376:1-8.

9. Nagao S, Kugita M, Yoshihara D, Yamaguchi T. Animal models for human polycystic kidney disease. Exp Anim. 2012;61:477-88.

10. Chen JR, Singhal R, Lazarenko OP, Liu X, Hogue WR, Badger TM, et al. Short term effects on bone quality associated with consumption of soy protein isolate and other dietary protein sources in rapidly growing female rats. Exp Biol Med (Maywood). 2008;233:1348-58.

11. Fernandes $G$, Lawrence $R$, Sun D. Protective role of $n-3$ lipids and soy protein in osteoporosis. Prostaglandins Leukot Essent Fatty Acids. 2003;68:361-72.

12. Lukas R, Gigliotti JC, Smith BJ, Altman S, Tou JC. Consumption of different sources of omega-3 polyunsaturated fatty acids by growing female rats affects long bone mass and microarchitecture. Bone. 2011:49:455-62.

13. Weiler HA, Kovacs H, Nitschmann E, Bankovic-Calic N, Aukema H, Ogborn M. Feeding flaxseed oil but not secoisolariciresinol diglucoside results in higher bone mass in healthy rats and rats with kidney disease. Prostaglandins Leukot Essent Fatty Acids. 2007;76:269-75.

14. Shah P, Mudaliar S. Pioglitazone: side effect and safety profile. Expert Opin Drug Saf. 2010;9:347-54.

15. Childs CE, Romeu-Nadal M, Burdge GC, Calder PC. Gender differences in the n-3 fatty acid content of tissues. Proc Nutr Soc. 2008;67:19-27.

16. Ogborn MR, Nitschmann E, Bankovic-Calic N, Weiler HA, Aukema HM. Effects of flaxseed derivatives in experimental polycystic kidney disease vary with animal gender. Lipids. 2006;41:1141-9.

17. NRC, National Research Council. Guide for the care and Use of laboratory animals. Washington, DC: National Academy Press; 1996.

18. NRC, National Research Council. Nutrient requirements of laboratory animals. Washington, DC: National Academy Press; 1995. 4th rev.

19. Cordain L, Eaton SB, Sebastian A, Mann N, Lindeberg S, Watkins BA, et al. Origins and evolution of the Western diet: health implications for the 21st century. Am J Clin Nutr. 2005;81:341-54.

20. Tou JC, Altman SN, Gigliotti JC, Benedito VA, Cordonier EL. Different sources of omega-3 polyunsaturated fatty acids affects apparent digestibility, tissue deposition, and tissue oxidative stability in growing female rats. Lipids Health Dis. 2011;10:179-85. 
21. Ammann P, Rizzoli R. Bone strength and its determinants. Osteoporos Int. 2003;14:S13-8.

22. Yuan $\mathrm{Y}$, Kitts DD. Estimation of dietary calcium utilization in rats using a biomechanical functional test. Food Chem. 1992:44:1-47.

23. Corey RL, Whitaker MD, Crowell MD, Keddis MT, Aqel B, Balan V, et al. Vitamin D deficiency, parathyroid hormone levels, and bone disease among patients with end-stage liver disease and normal serum creatinine awaiting liver transplantation. Clin Transplant. 2014;28:579-84.

24. Plischke $M$, Kohl M, Bankir L, Shayganfar S, Handisurya A, Heinze G, et al. Urine osmolarity and risk of dialysis initiation in a chronic kidney disease cohort-a possible titration target? PLoS One. 2014;9:e93226.

25. Maditz KH, Oldaker C, Nanda N, Benedito V, Livengood R, Tou JC. Dietary n-3 Polyunsaturated fatty acids or soy protein isolate did not attenuate disease progression in a female rat model of autosomal recessive polycystic kidney disease. Nutr Res. 2014;34:526-34.

26. Maditz KH, Benedito VA, Oldaker C, Nainika N, Lateef SS, Livengood R, et al. Feeding Soy Protein Isolate and Omega-3 Polyunsaturated Fatty Acids Affects Polycystic Liver Disease Progression in a PCK Rat Model of Autosomal Polycystic Kidney Disease. JPGN. (accepted).

27. Nickolas TL, Leonard MB, Shane E. Chronic kidney disease and bone fracture: a growing concern. Kidney Int. 2008;74:721-31.

28. Lu J, Bankovic-Calic N, Ogborn M, Saboorian MH, Aukema HM. Detrimental effects of a high fat diet in early renal injury are ameliorated by fish oil in Hans:SPRD-cy rats. J Nutr. 2003;133:180-6.

29. Ecder T. Cardiovascular complications in autosomal dominant polycystic kidney disease. Curr Hypertens Rev. 2003;9:2-11.

30. Cadnapaphornchai MA. Hypertension in children with autosomal dominant polycystic kidney disease (ADPKD). Curr Hypertens Rev. 2013;9:21-6.

31. Goto M, Hoxha N, Osman R, Dell KM. The renin-angiotensin system and hypertension in autosomal recessive polycystic kidney disease. Pediatr Nephrol. 2010;25:2449-57.

32. Shroff R. Dysregulated mineral metabolism in children with chronic kidney disease. Curr Opin Nephrol Hypertens. 2011;20:233-40.

33. Carney EF. Bone: cortical bone loss in patients with chronic kidney disease. Nat Rev Nephrol. 2013;9:248.

34. Norman LJ, Coleman JE, Macdonald IA, Tomsett AM, Watson A. Nutrition and growth in relation to severity of renal disease in children. Pediatr Nephrol. 2000;15:259-65.

35. Anastasia JV, Braun BL, Smith KT. General and histopathological results of a two-year study of rats fed semi-purified diets containing casein and soya protein. Food Chem Toxicol. 1990;28:147-56.

36. Sirois I, Cheung AM, Ward WE. Biomechanical bone strength and bone mass in young male and female rats fed a fish oil diet. Prostaglandins Leukot Essent Fatty Acids. 2003;68:415-21.

37. Aukema HM, Gauthier J, Roy M, Jia Y, Li H, Aluko RE. Distinctive effects of plant protein sources on renal disease progression and associated cardiac hypertrophy in experimental kidney disease. Mol Nutr Food Res. 2011;55:1044-51

38. Kuiper GG, Lemmen JG, Carlsson B, Corton JC, Safe SH, Van der Saag PT, et al. Interaction of estrogenic chemicals and phytoestrogens with estrogen receptor beta. Endocrinology. 1998;39:4252-63.

39. Chagin AS, Limber MK, Andersson N, Moverare S, Gustaftson JA, Savendahl $L$, et al. Estrogen receptor- $\beta$ inhibits skeletal growth and has the capacity to mediate growth plate fusion in female mice. J Bone Min Res. 2004;19:72-7.

40. Tou JC, Arnaud SB, Grindeland R, Wade C. The effect of purified compared with nonpurified diet on bone changes induced by hindlimb suspension of female rats. Exp Biol Med (Maywood). 2005;230:31-8.

41. Weiler H, Kovacs H, Nitschmann E, Wong SF, Bankovic-Calic N, Ogborn M. Elevated bone turnover in rat polycystic kidney disease is not due to prostaglandin $E_{2}$. Pediatr Nephrol. 2002;17:795-9.

42. Lane K, Derbyshire E, Li W, Brennan C. Bioavailability and potential uses of vegetarian sources of omega-3 fatty acids: a review of the literature. Crit Rev Food Sci Nutr. 2014;54:572-9.

43. Watkins BA, Li Y, Allen KG, Hoffmann WE, Seifert MF. Dietary ratio of $(n-6) /(n-3)$ polyunsaturated fatty acids alters the fatty acid composition of bone compartments and biomarkers of bone formation in rats. J Nutr. 2000;130:2274-84.

44. Watkins BA, Li Y, Lippman HE, Seifert MF. Omega-3 polyunsaturated fatty acids and skeletal health. Exp Biol Med (Maywood). 2001;226:485-97.

45. Decsi T, Kennedy K. Sex-specific differences in essential fatty acid metabolism Am J Clin Nutr. 2011;94(suppl):1914S-9.
46. Reeves PG. Components of the AIN-93 diets as improvements in the AIN-76A diet. J Nutr. 1997;127:suppl:8385-8841.

47. Masyuk TV, Huang BQ, Masyuk Al, Ritman EL, Torres VE, Wang X, et al. Biliary dysgenesis in the PCK rat, an orthologous model of autosomal recessive polycystic kidney disease. Am J Pathol. 2004;165:1719-30.

48. Guenther PM, Casavale KO, Reedy J, Kirkpatrick SI, Hiza HA, Kuczynski KJ, et al. Update of the healthy eating index: HEl-2010. J Acad Nutr Diet. 2013;113:569-80.

\section{Submit your next manuscript to BioMed Central and take full advantage of:}

- Convenient online submission

- Thorough peer review

- No space constraints or color figure charges

- Immediate publication on acceptance

- Inclusion in PubMed, CAS, Scopus and Google Scholar

- Research which is freely available for redistribution 